\title{
Graded Control Technology of Oil Stability and Water Control in Fracture-Pore Carbonate Reservoirs
}

\author{
Wenqi Zhao $\mathbb{D}^{1},{ }^{1}$ Lun Zhao $\mathbb{D}^{1},{ }^{1}$ Junjian $\mathrm{Li}^{2}{ }^{2}$ Jue Hou, ${ }^{1}$ and Xuejing Guo ${ }^{1}$ \\ ${ }^{1}$ PetroChina Research Institute of Petroleum Exploration \& Development, China National Petroleum Corporation, \\ Beijing 100083, China \\ ${ }^{2}$ China University of Petroleum, Beijing 102249, China
}

Correspondence should be addressed to Lun Zhao; zhaolun@petrochina.com.cn

Received 18 November 2021; Accepted 17 January 2022; Published 8 February 2022

Academic Editor: Xiang Zhou

Copyright (c) 2022 Wenqi Zhao et al. This is an open access article distributed under the Creative Commons Attribution License, which permits unrestricted use, distribution, and reproduction in any medium, provided the original work is properly cited.

In view of the prominent problems of extremely strong heterogeneity, fracture development of different scales, and serious rapid water channelling in the process of water injection development in fracture-pore carbonate reservoirs, a comprehensive treatment method of oil stabilizing and water controlling is proposed, which includes fracture classification control, combination of adjustment and plugging, and wettability change. Based on the static and dynamic test data, the characteristics of reservoir fracture development and fracture scales are described in detail. By means of reservoir engineering and numerical simulation, the water flooding rule under different grade fracture reservoir conditions is studied, the influence of different levels of fractures on water injection development is evaluated, and the improvement effect of different control methods on water drive sweep is demonstrated. Research shows that a large amount of remaining oil trapped in pores is affected by fracture water channelling, which makes it difficult to use effectively. The water flooding sweep characteristics of reservoirs with different scales of fractures are quite different, and large-scale fractures are the main reason for ineffective water injection. The hierarchical control techniques of plugging large-scale fractures, intervening and adjusting small- and medium-scale fractures, and changing the wettability of microscale pore media can significantly extend the water flooding spread range and effectively improve oil well production. The research results have been applied in the field and achieved good results. This technology can provide theoretical guidance and a technical basis for the comprehensive treatment of oil stabilization and water control in fractured-porous carbonate oil and gas reservoirs.

\section{Introduction}

As one of the most important oil and gas reservoirs globally, carbonate reservoirs account for an increasing proportion of oil and gas reserves and production. However, carbonate reservoirs are complex, bringing difficulties in hydrocarbon exploitation. Carbonate reservoir development technology has become a challenge and an important research area in global and domestic oil development [1-5]. To date, experiences in developing complex carbonate reservoirs are limited both at home and abroad [6-9]. Carbonate reservoirs have various types of pores, mainly including large-scale cavities, small-scale pores, and multiscale fractures. Fractured carbonate reservoirs commonly have internal preferential pathways, resulting in severe water channelling problems and poor development results. Consequently, oil stabilization and water control are essential for the oil recovery of fractured carbonate reservoirs. Reservoir development practice at home and abroad shows that plugging technology is an essential means to improve water drive effect. The injection of chemical agents to block the dominant flow channel can adjust the liquid production profile, alleviate the internal contradictions in the reservoir, and improve the effect of oilfield development, but the plugging technology of carbonate reservoirs is not mature enough. The existing methods are difficult to implement and cannot truly combine the real geological characteristics of the oilfield. The process and selection of chemicals are relatively simple, and the reservoir is only generally blocked at the macrolevel. Therefore, the previous technology cannot really 
play profile control water, alleviating reservoir contradictions. It is difficult to achieve hierarchical control and achieve the desired development effect.

This paper takes the fractured-porous carbonate reservoirs in the $\mathrm{T}$ oilfield at the eastern margin of the PreCaspian Basin as an example to study the characteristics of fractures and the principle of water flooding in different levels of fractured reservoirs. The purpose is to propose a graded regulation method for stabilizing oil and controlling water in fractured-porous carbonate reservoirs. This proposed method can effectively block the large-scale media to avoid the rapid influx of injected water, effectively intervene the medium- and small-scale media to inhibit the seepage velocity of injected water, and improve the rock wettability of microscale media to improve the water drive efficiency. The suggested method is closely combined with the reservoir geological characteristics and development law and has stronger pertinence. It can effectively improve the production and absorption profile, expand the swept volume of water flooding, and improve the macroscopic swept range and microscopic oil displacement efficiency. This has important practical significance for improving oilfield development effects.

\subsection{Geological Characteristic of Carbonate Reservoirs.} Structures of the Pre-Caspian Basin can be found in Figure 1. More than $90 \%$ of the oil and gas reserves in the Pre-Caspian Basin are from carbonate reservoirs. The strata of the basin can be divided into two sets, which are above-salt and subsalt strata. All currently discovered carbonate reservoirs are located below the salt layer. Two oil-bearing reservoirs, Carboniferous KT-I and KT-II, are developed. Lithology at the eastern margin of the basin is complex [10-14]. Limestone and dolomite are dominated by minor gypsum and others.

The carbonate reservoirs in the Pre-Caspian Basin are influenced by sedimentary, diagenetic, and tectonic processes, resulting in various reservoir space types, such as pores, fractures, and caves. Based on the observation of cores, thin sections, and scanning electron microscopic photos, it shows that pores are the dominant oil accumulation space. The volume of the pores accounts for $94 \%$ of the total volume of the space. Fractures and caves are $5 \%$ and $1 \%$, respectively. Large-scale fractures and cavities are relatively abundant.

According to the dip angle, the fractures can be divided into low angle, oblique, and high angle, as shown in Table 1. Based on core and image logging data, the number of different types of fractures is counted. The statistical results are shown in Figure 2. The results show that the low-angle fractures account for $80.5 \%$ and $57.5 \%$ in core and imaging logging data. Oblique fractures account for $14.6 \%$ and $35.8 \%$ in core and imaging logging data, and high-angle fractures account for $4.9 \%$ and $6.7 \%$ in core and image logging data. In general, low-angle fractures are dominated.

According to the opening size, the fractures are divided into large-scale fractures, mesoscale fractures, small-scale fractures, and microscale fractures, as shown in Table 2. The number of fractures with different opening sizes is counted based on imaging logging, as shown in Figure 3. The results show that the proportions of large-scale, mesoscale, smallscale, and microscale fractures in the reservoir are $4.7 \%$, $52.5 \%, 42.5 \%$, and $0.4 \%$, respectively. In general, mesoscale and small-scale fractures are dominated.

The types of reservoir accumulation space and space combination are complex. According to the development degree and space combination, the reservoir space can be divided into five types based on core observation and well logging response. The five types of reservoir space include the porous-cavernous-fractured composite type, porouscavernous type, porous type, fractured-porous type, and fractured type. The proportions of each type are $6.2 \%, 5.3 \%$, $44.9 \%, 42.6 \%$, and $1.0 \%$, respectively. In general, the fractured pore type is dominated.

\section{Seepage Characteristics of Water Injection in Different Types of Reservoirs}

In order to better understand the effect of water flooding, seepage characteristics of water injection in reservoirs with fractured, fractured-porous, and porous-cavernous spaces are discussed by using microfluidic technology [15-18]. The first step is to select the typical thin section that represents various types of reservoirs and design a model based on pore-throat structure extraction and image stitching, as shown in Figure 4. Then, the microfluidic water drive experiment is carried out to analyze the water injection seepage characteristics of different types of reservoirs. During the microfluidic experiment, the thin section is first injected with kerosene for saturation, and then, at one end of the thin section, water is injected at an injection rate of $1 \mu \mathrm{L} / \mathrm{min}$ to displace kerosene, and the other end is the production end. During the experiment, the displacement process is recorded by using a high-power camera.

Analyzing the thin reservoir section with the pore space type shows that the maximum, minimum, and average pore sizes are $613 \mu \mathrm{m}, 6.1 \mu \mathrm{m}$, and $63.4 \mu \mathrm{m}$, respectively. The main pore size distribution is below $200 \mu \mathrm{m}$. The porous reservoir is heterogeneous, which can be observed from the water flooding test of the oil-saturated thin section, as shown in Figure 5. The injected water is relatively fully affected by the thin section when it enters the pores and throats. The front of the injected water is relatively uniform. The flooded water has a wider sweep area and low penetration speed. The flooding effect is mainly affected by the pore size, pore distribution, and connectivity of the pore and throat. The recovery rate of this model is $43.7 \%$.

Compared with the porous reservoir, the fracturedporous reservoir is more heterogeneous. The maximum, minimum, and average pore sizes are $754 \mu \mathrm{m}, 10.2 \mu \mathrm{m}$, and $122.9 \mu \mathrm{m}$, respectively. The main pore size distribution is below $250 \mu \mathrm{m}$. The opening degree of fractures is different in various places, enhancing the heterogeneity of the reservoir. It can be observed from the water flooding test of the oilsaturated thin section, as shown in Figure 6. The injected water first enters the matrix around the injection point and then the fractures. Finally, the injected water rapidly 


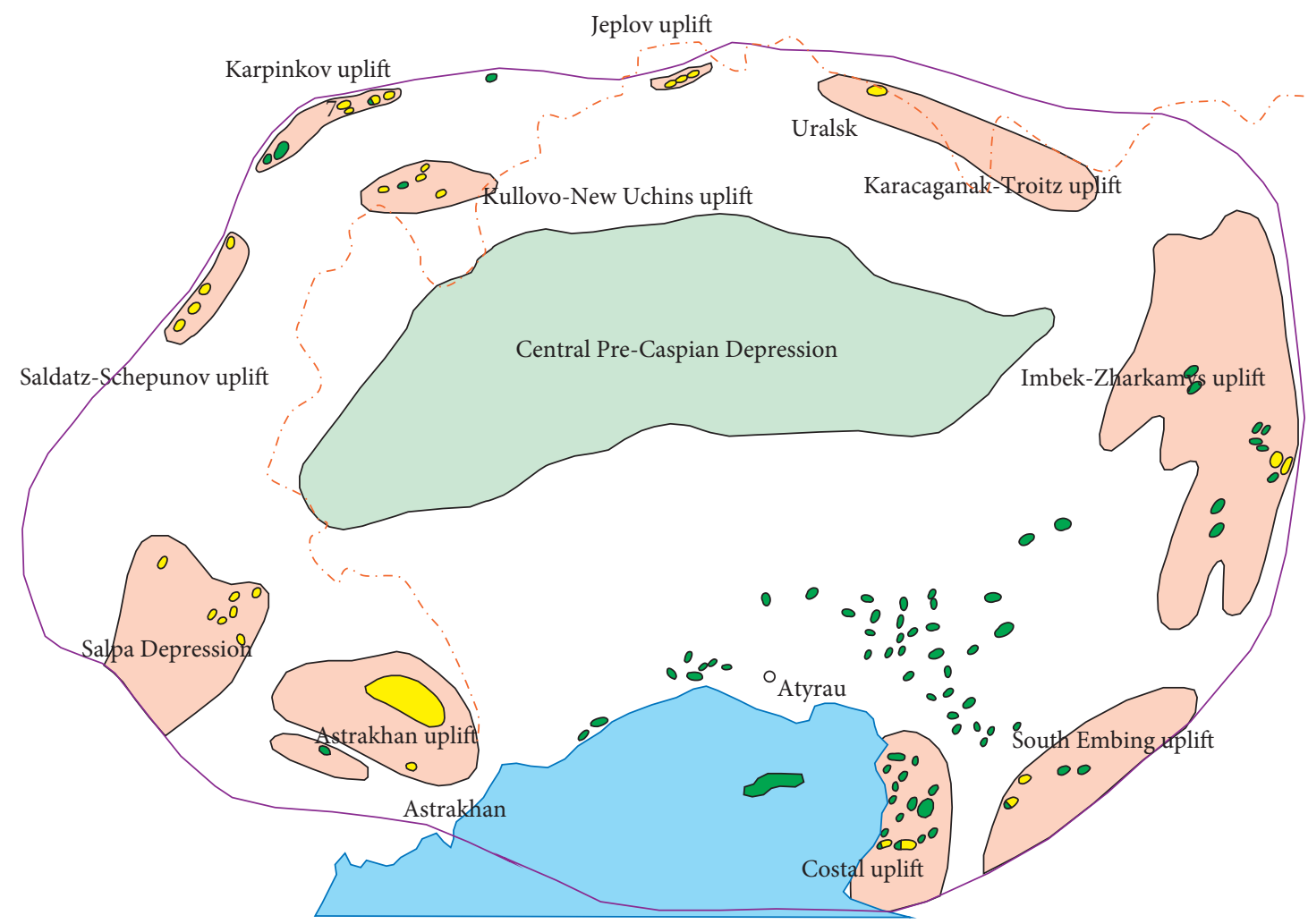

Figure 1: Structures of the Pre-Caspian Basin.

TABLE 1: Fracture dip angle and fracture types.

\begin{tabular}{lccc}
\hline Fracture dip angle & $>70^{\circ}$ & $35^{\circ}-70^{\circ}$ & $<35^{\circ}$ \\
\hline Fracture type & High-angle fracture & Oblique fracture & Low-angle fracture \\
\hline
\end{tabular}

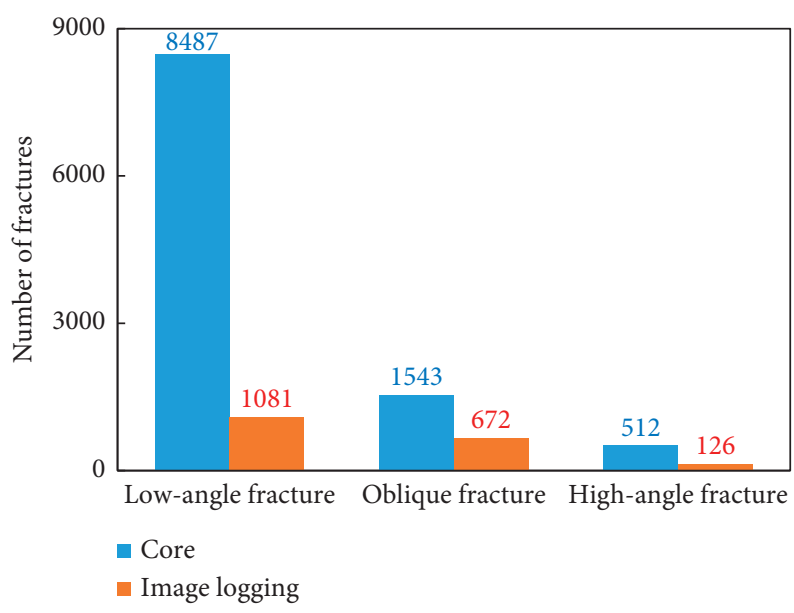

Figure 2: Numbers of the fractures with different types.

TABle 2: Fracture opening size and fracture type.

\begin{tabular}{lcccc}
\hline Fracture opening size $(\mathrm{mm})$ & $<0.001$ & $0.001-0.01$ & $0.01-0.1$ & $>0.1$ \\
\hline Fracture type & Microscale & Small-scale & Mesoscale & Large-scale \\
\hline
\end{tabular}




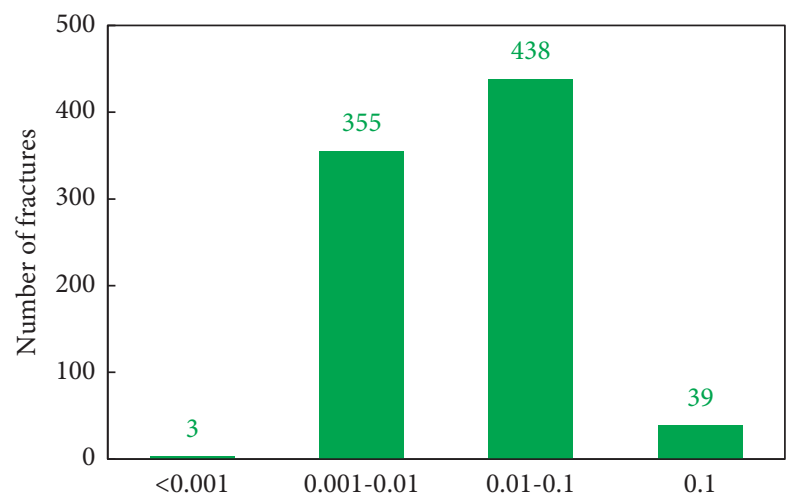

FIGURE 3: Numbers of the fractures with different opening sizes.

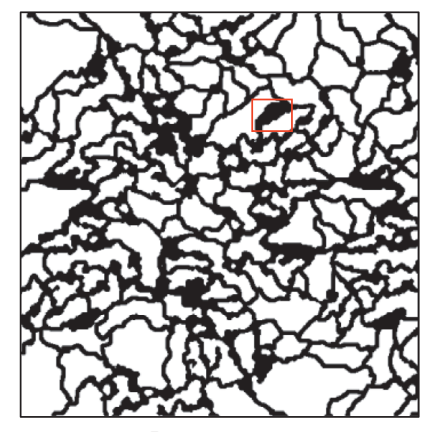

Porous type

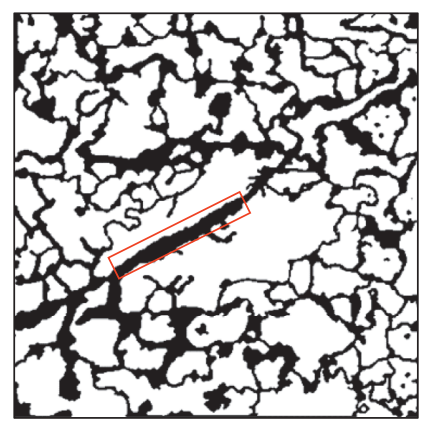

Fractured porous type

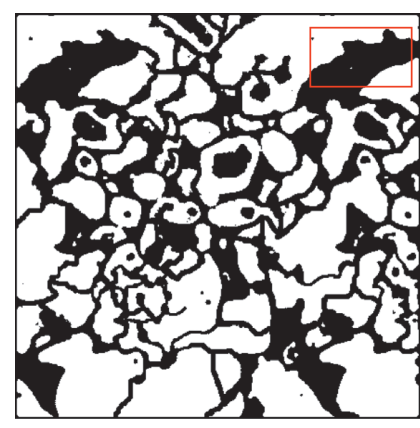

Porous-cavernous type

Figure 4: Experimental models of microfluid in different space types of reservoirs (pores, fractures, and caves are marked in the red box). (a) Porous type. (b) Fractured-porous type. (c) Porous-cavernous type.
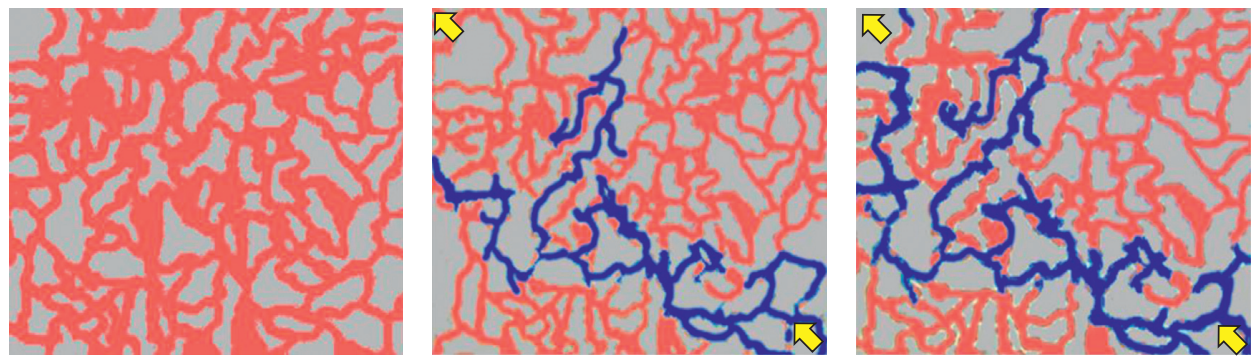

FIGURE 5: Schematic diagram of the early, middle, and late stages of water injection in porous reservoirs.
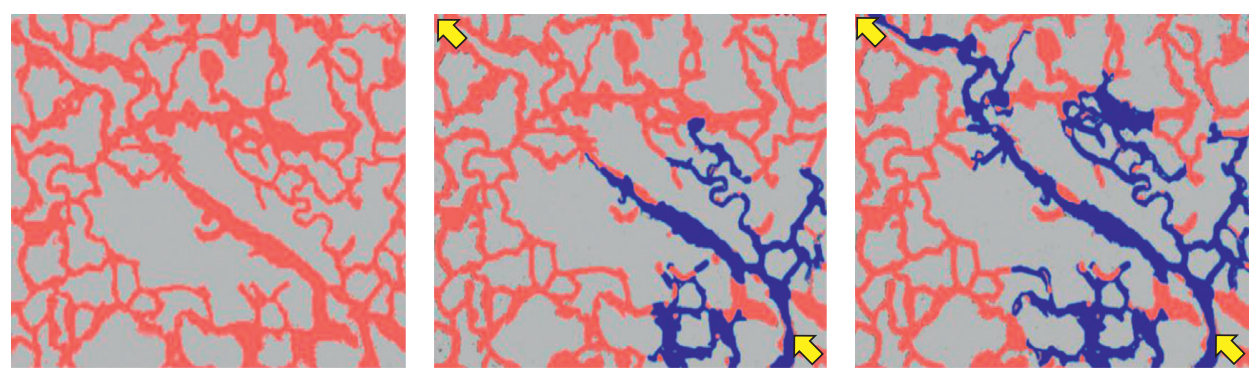

FIGURE 6: Schematic diagram of the early, middle, and late stages of water injection in fractured-porous reservoirs.

advances along the cracks. Simultaneously, part of the injected water sweeps the surrounding areas with better connectivity. After the water breakthrough, the scope of the injected water does not increase. The oil in the fractures is effectively displaced, whereas the matrix has a poorer oil displacement effect. The recovery rate of this model is $35.4 \%$. 
The reservoir with the porous-cavernous space type also shows strong heterogeneity. The maximum, minimum, and average pore sizes are $1031 \mu \mathrm{m}, 13.1 \mu \mathrm{m}$, and $123.7 \mu \mathrm{m}$, respectively. The main pore size distribution is below $550 \mu \mathrm{m}$. The injected water easily enters the large cavities and gradually displaces the oil from the cavities to the surrounding pores with good connectivity. This can be observed from the water flooding test of the oil-saturated thin section, as shown in Figure 7. The swept area of the injected water decreases. The cavities mainly play a role in storage. The water flooding effect is mainly affected by the degree of connectivity between the cavities and the throats. The recovery rate of this model is $38.6 \%$.

From the above analyses, it can be seen that the water flooding effects of different types of reservoirs are different. Due to the presence of fractures and cavities, the injected water flows fast, and the swept area is small. Therefore, the fractures and cavities determine the effect of water flooding development. For the T oilfield, cavities are relatively small. Consequently, fractures are the key to stabilizing oil and controlling water in the T oilfield.

In order to better understand the role of fractures in the water flooding process, digital core simulation methods were used to simulate the porous, fractured-porous, and fractured reservoirs [19-23], as shown in Figure 8. The results show that the development of fractures is the main factor influencing the effect of water flooding. Table 3 shows that the water flood recovery rates of fractured and fractured-porous reservoirs are around 17\%, significantly lower than the $40 \%$ recovery rate of porous reservoirs. The remaining movable oil in fractured-porous reservoirs is significantly higher than that in porous reservoirs. Therefore, improving the effectiveness of water flooding in fractured reservoirs is the direction of adjustment of oilfield water injection development.

\section{Graded Water Flooding Control of Fractured Reservoirs with Variable Opening Sizes}

3.1. Water Flooding Effect of Fractures with Different Opening Sizes. The porosity and permeability of 1991 cores from 10 wells in the study area were measured by using an overburden pressure porosity and permeability tester to simulate formation conditions, including 171 fractured cores and 1820 nonfractured cores. Based on the analysis of the relationship between porosity and permeability of cores, it can be seen that fractures can significantly improve the permeability of the reservoir, especially when the porosity is less than $10 \%$, as shown in Figure 9. The fractures can increase the permeability of the reservoir by 10 to 100 times.

Although fractures can improve the conductivity of fluids in the reservoir, the injected water easily flows along with the fractures, resulting in a large amount of oil being immobilized in the matrix and unable to be recovered. In order to better understand the role of fractures with different opening sizes in the water flooding process, the embedded discrete fracture numerical simulation method is used to analyze the sweep characteristics of water flooding. The fracture opening sizes are designed to be $0.001 \mathrm{~mm}$,
$0.005 \mathrm{~mm}, 0.01 \mathrm{~mm}, 0.05 \mathrm{~mm}, 0.1 \mathrm{~mm}$, and zero, as shown in Figure 10. Under the condition of 0.4 times the pore volume, when water drive development is carried out in the reservoir without fractures and with a fracture opening of $0.001 \mathrm{~mm}$, the injected water advances more evenly, the water drive front edge is relatively flat, no obvious water channelling hardly occurs, and the water drive has a wide range. When the opening size is greater than $0.005 \mathrm{~mm}$, water channelling occurs. There is a tongue at the front edge of the water drive, and the fracture begins to affect the water flow velocity, which has an adverse impact on the effect of water injection development. It shows that the fracture improves the conductivity of the reservoir, and with the increase of opening, the conductivity of the reservoir increases, and the channelling speed of injected water becomes faster. The water channelling is obvious when the opening size is $0.1 \mathrm{~mm}$, and the swept area of water flooding is limited. The difference of permeability grade between the fracture and the matrix becomes larger, and the injected water rapidly rushes into the production end along the fracture, resulting in poor water drive development effect.

As is shown in Figure 11, the water flooding sweep coefficient of the nonfracture model can reach $62.5 \%$. With the increase of the fracture opening size, the water flooding sweep coefficient gradually decreases. When the opening size increases to over $0.01 \mathrm{~mm}$, the water flooding sweep coefficient decreases significantly. When the fracture opening size is $0.1 \mathrm{~mm}$, the water flooding sweep coefficient is only $31.3 \%$. Therefore, the water flooding effect of fractures with variable opening sizes is different. When the fracture opening size is under $0.001 \mathrm{~mm}$, there is no obvious penetration of the injected water. When the fracture opening size reaches more than $0.01 \mathrm{~mm}$, the water injection effect starts to deteriorate significantly. When the reservoir has large-scale fractures with an opening size of more than $0.1 \mathrm{~mm}$, the injected water shows obvious channelling, which easily leads to ineffective water injection.

3.2. Profile Control of Fractures with Different Opening Sizes. According to the aforementioned research results, the water flooding features of fractured reservoirs of variable opening sizes are different. The roles of fractures with variable opening sizes played in water injection development are also different. Therefore, it is necessary to conduct different order control on fractures to understand the effectiveness of water injection. The numerical simulation model of fractured reservoirs with fracture openings of $0.1 \mathrm{~mm}$ (large-scale), $0.01 \mathrm{~mm}$ (medium- to small-scale), and $0.001 \mathrm{~mm}$ (microscale) is designed. Flexible gels, nano-microspheres, and wettability modifiers are used to inject into reservoir samples at 0.5 times the fracture volume, 0.5 times the fracture volume, and 0.2 times the matrix volume, respectively. Then, samples are flooded with water. The concentration of modifiers in fractured reservoirs with variable opening sizes and the oil saturation after adjustment are shown in Figures 12-14.

Based on the distribution pattern of the chemical agent after the flexible gel flooding, it shows that, as the fracture 

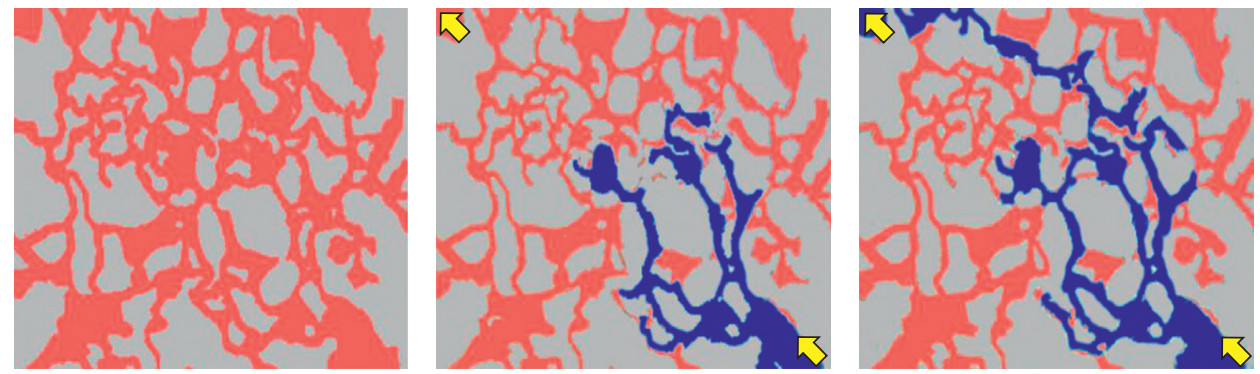

FIGURE 7: Schematic diagram of the early, middle, and late stages of water injection in a porous-cavernous reservoir.

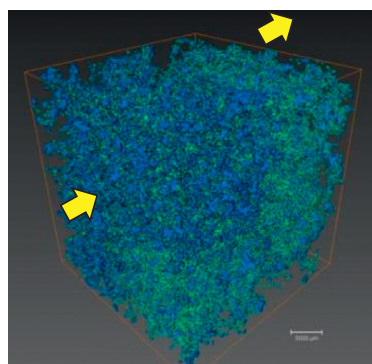

Porous type

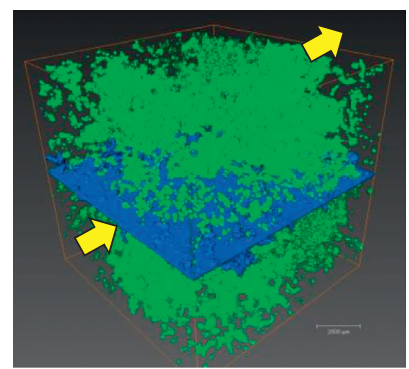

Fractured-porous type

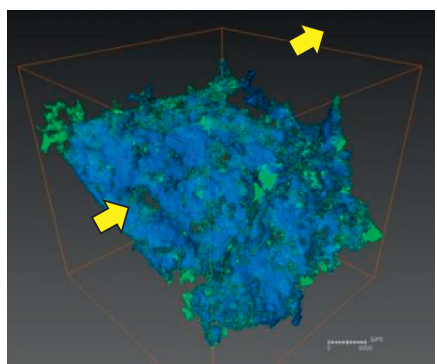

Porous-cavernous type

Figure 8: Digital core simulation of movable remaining oil distribution in different types of reservoirs. (a) Porous type. (b) Fracturedporous type. (c) Porous-cavernous type.

TABLE 3: Porosity and remaining oil saturation of different types of reservoirs.

\begin{tabular}{|c|c|c|c|c|c|c|c|}
\hline Reservoir type & $\begin{array}{c}\text { Total } \\
\text { porosity (\%) }\end{array}$ & $\begin{array}{c}\text { Connected } \\
\text { porosity (\%) }\end{array}$ & $\begin{array}{c}\text { Effective } \\
\text { porosity (\%) }\end{array}$ & $\begin{array}{c}\text { Irreducible water } \\
\text { saturation (\%) }\end{array}$ & $\begin{array}{c}\text { Residual oil } \\
\text { saturation (\%) }\end{array}$ & $\begin{array}{c}\text { Movable remaining } \\
\text { oil saturation (\%) }\end{array}$ & $\begin{array}{l}\text { Recovery } \\
\text { rate }(\%)\end{array}$ \\
\hline Porous type & 12.47 & 10.81 & 5.39 & 32.02 & 24.72 & 16.05 & 40 \\
\hline Fractured type & 5.25 & 1.43 & 0.54 & 49.40 & 40.28 & 1.66 & 17.1 \\
\hline $\begin{array}{l}\text { Fractured- } \\
\text { porous type }\end{array}$ & 13.48 & 11.82 & 4.13 & 38.04 & 31.34 & 20.13 & 16.9 \\
\hline
\end{tabular}

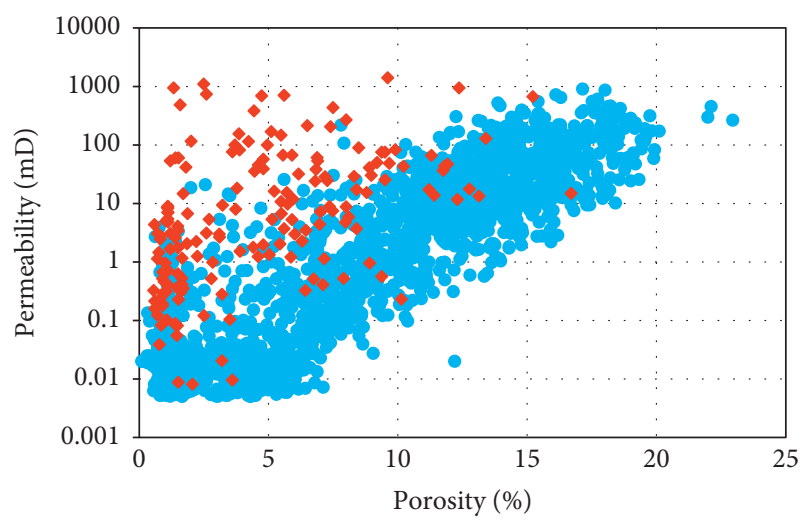

- No fracture

- With fracture

FIGURE 9: The relationship between porosity and permeability of cores.

size and profile depth decrease, the sweep coefficient of large-scale, medium-small-scale, and microscale fractured reservoirs increases to $11.4 \%, 5.2 \%$, and $2.5 \%$, respectively.
The large-scale fracture has the largest increase in the sweep coefficient. The large-scale fractures can achieve deep profile control and flooding. The water channelling problem is controlled after the fractures are deeply controlled.

Based on the distribution pattern of the chemical agent after the nano-microsphere controlling and flooding, it shows that nano-microspheres can migrate into large-scale and medium-small-scale fractures. In microscale fractured reservoirs, the chemical agent is concentrated around the injection well. After nano-microsphere profile control and flooding, the large-scale, medium-small-scale, and microscale fractured reservoir sweep coefficients are increased by $4.8 \%, 8.5 \%$, and $2.5 \%$, respectively. The sweep coefficient of the small-medium-scale fractured reservoir has the largest increase. Although nano-microspheres can realize deep adjustment and flooding of large-scale fractures, they have limited fracture control. They cannot effectively control the channelling of injected water in large-scale fractures. For small- and medium-scale fractured reservoirs, nano-microspheres can achieve deep adjustment and flooding and obvious control over medium-small-scale fractures and uniform water flooding results. 


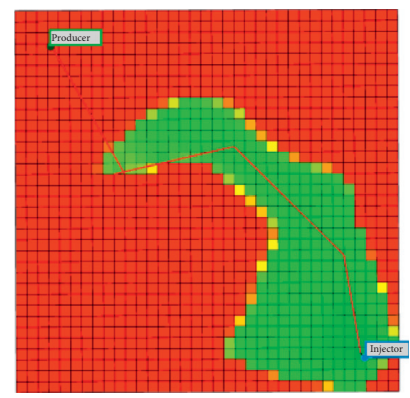

$0.1 \mathrm{~mm}$ opening size

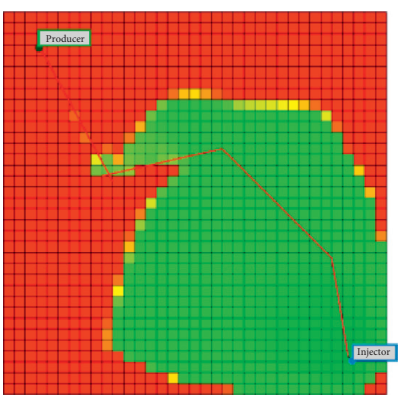

$0.005 \mathrm{~mm}$ opening size

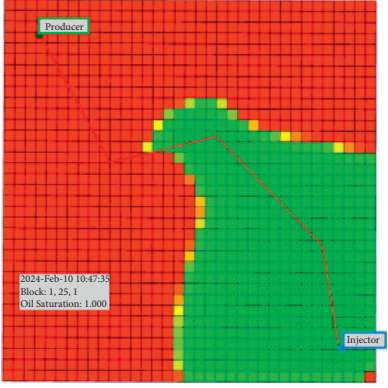

$0.5 \mathrm{~mm}$ opening size

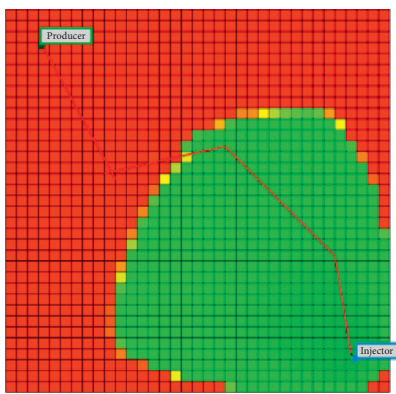

$0.001 \mathrm{~mm}$ opening size

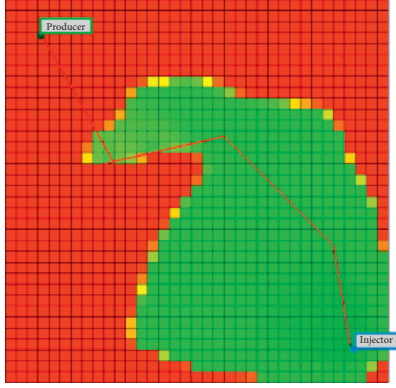

$0.01 \mathrm{~mm}$ opening size

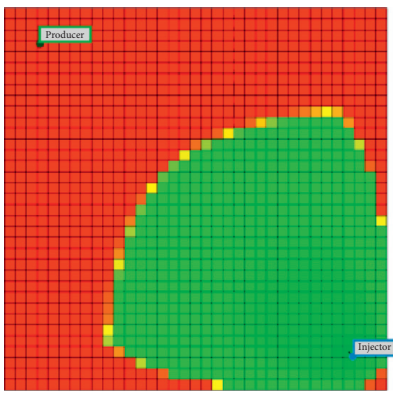

No fracture

FIGURE 10: Oil saturation distribution of water flooding in reservoirs with different fracture openings. (a) $0.1 \mathrm{~mm}$ opening size. (b) $0.5 \mathrm{~mm}$ opening size. (c) $0.01 \mathrm{~mm}$ opening size. (d) $0.005 \mathrm{~mm}$ opening size. (e) $0.001 \mathrm{~mm}$ opening size. (f) No fracture.

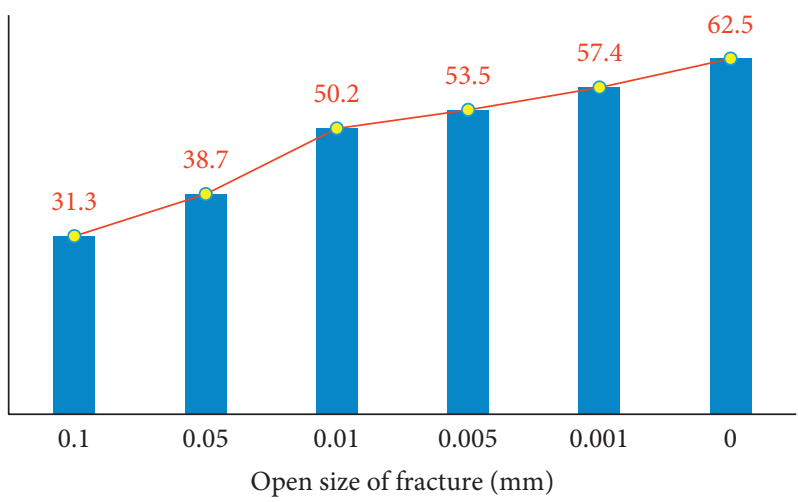

- Sweep coefficient $(\%)$

FIGURE 11: Water flooding sweep coefficients of fractured reservoirs with variable opening sizes.

Based on the distribution pattern of the surfactant injection, it shows that the surfactant can migrate into the fractures with different scales. In the microscale fractures, the agent is relatively enriched around the injection well. After surfactant injection, the large-scale, medium-smallscale, and microscale fractured reservoir sweep coefficients are increased by $4.8 \%, 8.5 \%$, and $2.5 \%$, respectively. The sweep coefficient of the microscale fractured reservoir has the largest increase. Surfactants can effectively improve the wettability of rocks in microscale fractured reservoirs. The oil in the matrix pores can be recovered, significantly improving the water flooding effect of microscale fractured reservoirs.

From the above analysis, it can be seen that the control methods of fractured reservoirs with variable fracture sizes are different. Large-scale, small-medium-scale, and microscale fractured reservoirs can be controlled with flexible gels, nano-microspheres, and surfactants to achieve better water flooding results.

\subsection{Control Method for Oil Stabilization and Water Control.} The research mentioned above shows that low angle is mainly developed in the study area. Water channelling easily occurs during water injection. The direction of water flooding is complicated, which seriously reduces oil production. Therefore, effective control of fractures is the key to stabilizing oil and controlling water in fractured-porous carbonate reservoirs. Fractures with variable scales are developed in the oilfield. The effects of fractures with different 

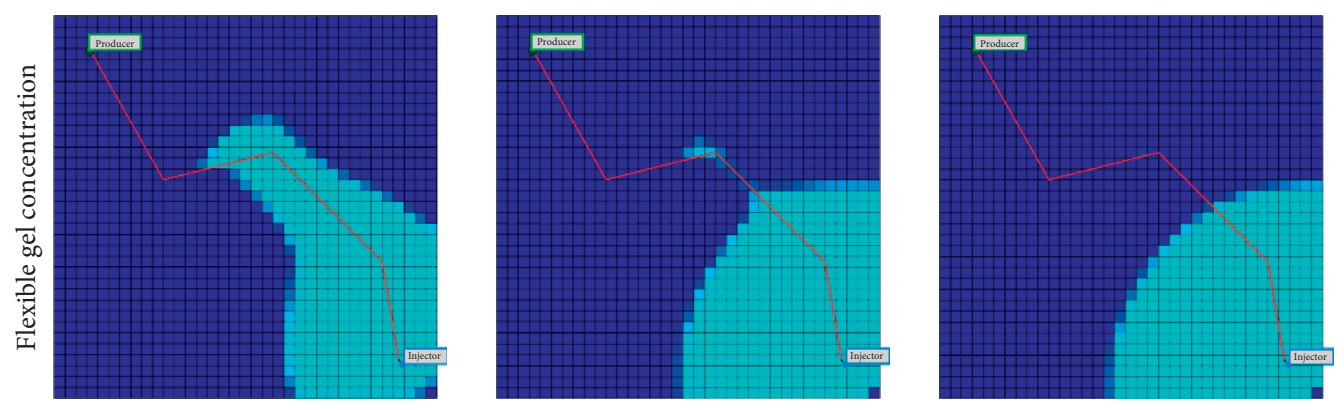

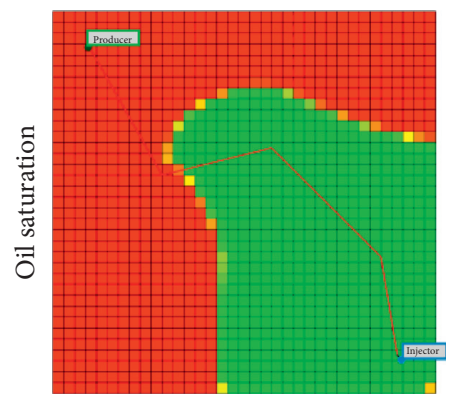

$0.1 \mathrm{~mm}$ opening size

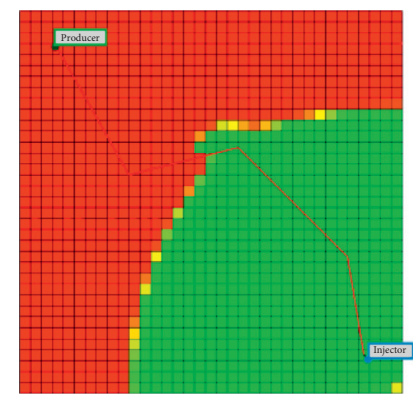

$0.01 \mathrm{~mm}$ opening size

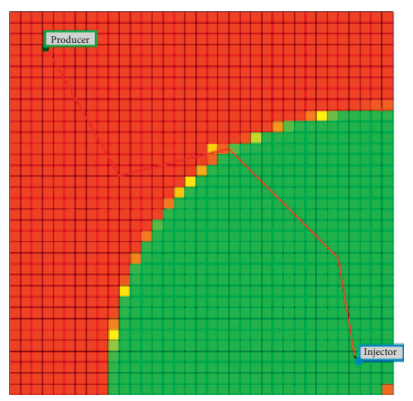

$0.001 \mathrm{~mm}$ opening size

FIGURE 12: Distribution of chemical agent concentration and oil saturation after control and flooding with flexible gel in fractured reservoirs with variable opening sizes.
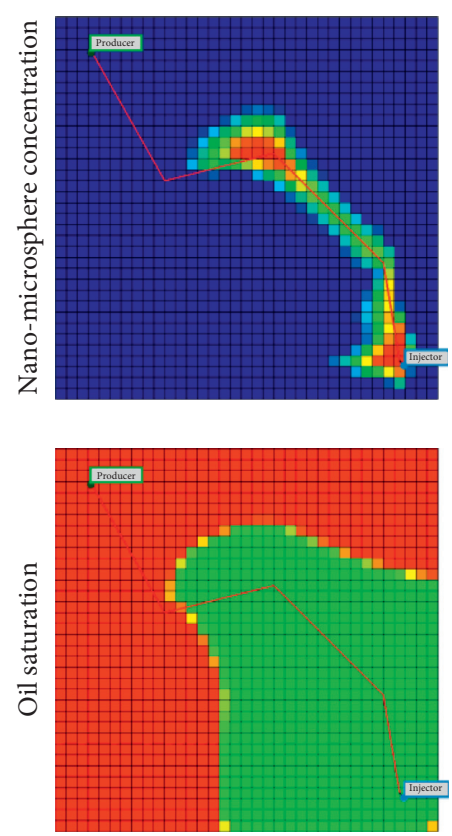

$0.1 \mathrm{~mm}$ opening size
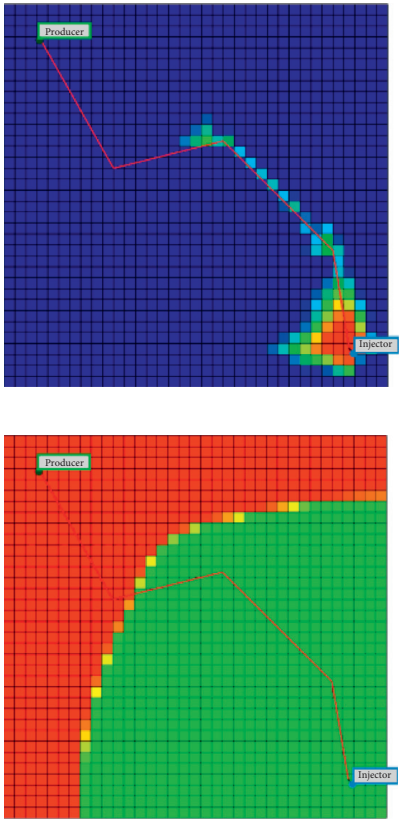

$0.01 \mathrm{~mm}$ opening size
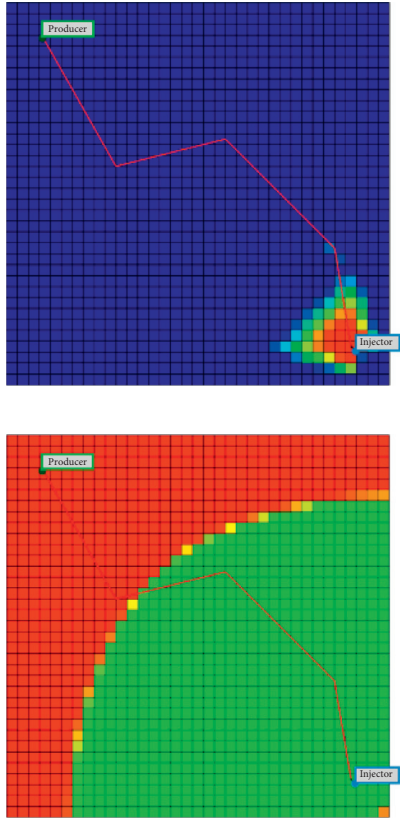

$0.001 \mathrm{~mm}$ opening size

FIGURE 13: Chemical agent concentration and oil saturation distribution after control and flooding with nano-microspheres in fractured reservoirs with variable opening sizes.

scales in the water flooding are quite different. Different control strategies should be adopted for different scale fractures based on the profile control results of variable fractures.

Large-scale fractures are the main channel for the rapid penetration of injected water, which can easily cause ineffective water injection. Large-scale fractures need to be effectively plugged and effectively solve the channelling problem. Small-medium-scale fractures are secondary channels for injected water intrusion. The flow rate of injected water is relatively fast, which largely affects the effect of water injection. Effective intervention needs to be taken to suppress the seepage rate of injected water. Microscale fractures and matrices are the main oil accumulation areas, 

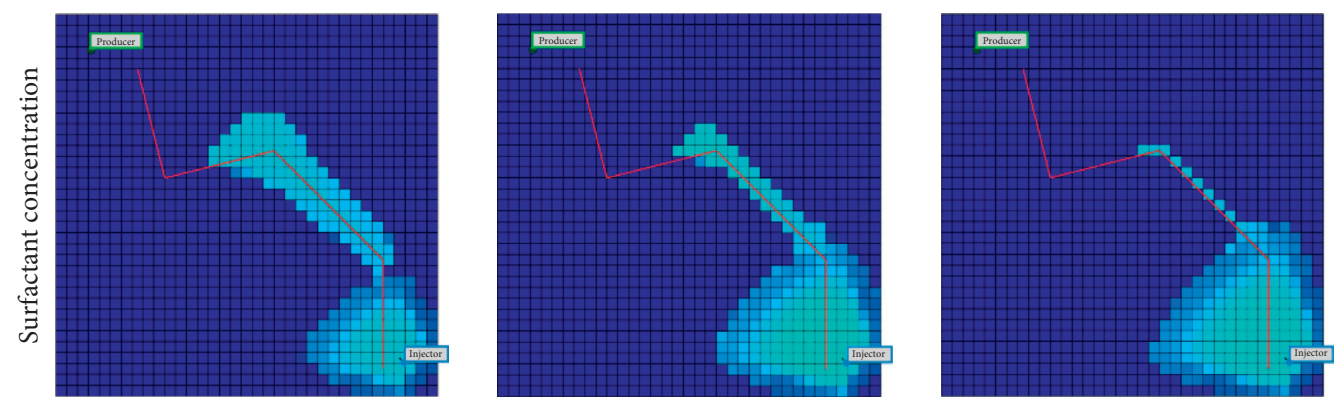

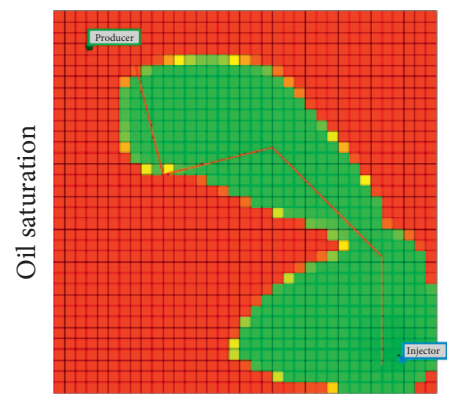

$0.1 \mathrm{~mm}$ opening size

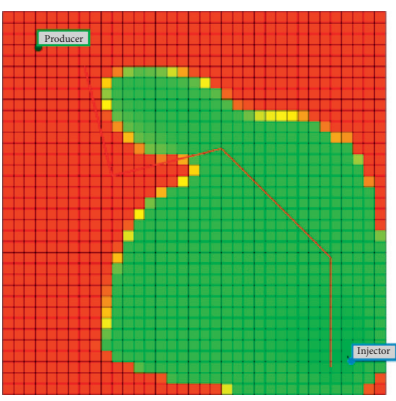

$0.01 \mathrm{~mm}$ opening size

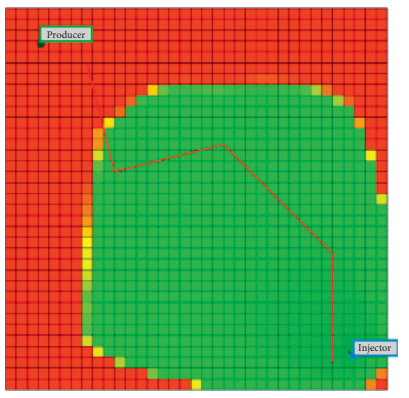

$0.001 \mathrm{~mm}$ opening size

FIGURE 14: Distribution of chemical agent concentration and oil saturation after surfactant injection in fractured reservoirs with variable opening sizes.

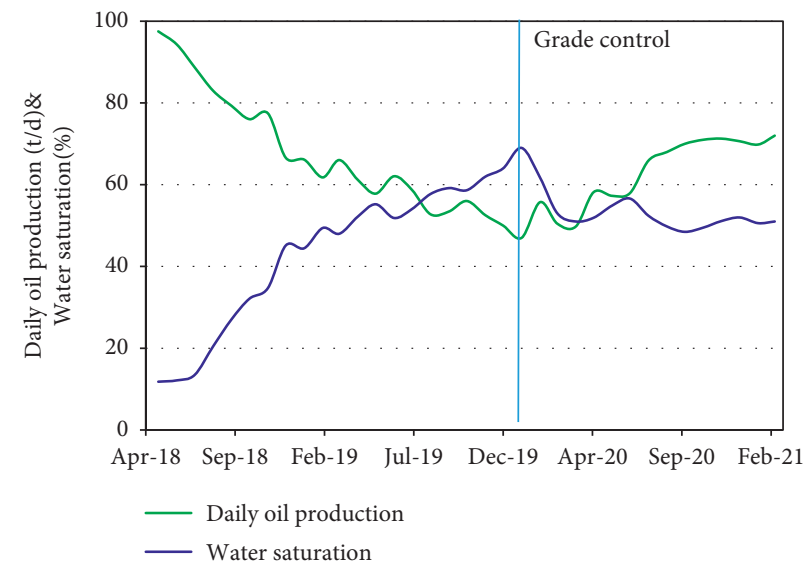

FIGURe 15: Production performance of wells in the pilot test area.

which are key to enhancing productivity. The wettability of rocks needs to be improved to increase water flooding efficiency.

Due to the presence of multiscale fractures in the reservoir, it is necessary to perform hierarchical control on fractures with different scales and use multiple control agents and slugs for comprehensive intervention in order to form a grading control method for oil stabilization and water control. The first plug uses flexible gel to block large-scale fractures. The second plug uses a nano-microsphere to adjust smallmedium-scale fractures. The third plug uses surfactant to change the wettability of the microscale pore and block the channelling. The purpose is to adjust the secondary preferential channel and to reduce the flow resistance of the matrix. Consequently, macroswept volume is expanded. The microdisplacement efficiency is improved. Finally, the goal of oil stabilization and water control can be achieved.

\section{Application Result}

The technology was carried out in January 2020 in the T oilfield at the eastern margin of the Pre-Caspian Basin. The graded control technology has been implemented in four water injection wells and ten surrounding oil wells. As depicted in Figure 15, the development effect has been significantly improved. The oil production increases $4 \mathrm{t} / \mathrm{d}$ based on the calculation result of the declining method. The water cut has dropped by $18 \% .279$ tonnes of oil will be increased. Due to the good performance, it is ready to expand the test scale within the entire oilfield. 
China operates about 20 carbonate oil reservoirs in Central Asia. This technology can be applied to the development of carbonate hydrocarbon reservoirs in Kazakhstan, providing technological support for enhancing oil recovery in Central Asia. To date, experiences in the development of complex carbonate reservoirs through water injection are limited both at home and abroad. This technology can be used for Sino-foreign cooperation projects in Kazakhstan, Oman, Turkmenistan, Iraq, and Syria, making a contribution to oil stabilization and water control in complex carbonate oil and gas fields.

\section{Conclusions}

This paper reveals the seepage characteristics of different types of reservoirs and factors affecting oil development based on the reservoir characteristics, water flooding principles, physical simulation, and digital core displacement experiment. The purpose is to understand the role of fractures in water flooding and the water flooding effect after fracture adjustment. Finally, graded control technology is proposed for oil stabilization and water control in fractured reservoirs with variable scale fractures. The specific conclusions are as follows:

(1) Reservoir types in the study area are diverse. The seepage characteristics of different types of reservoirs are different. The seepage rate of injected water in porous reservoirs is relatively slow-the water flooding front advances more uniformly. The swept area is wide. The water flooding development effect is good.

(2) The presence of fractures enhances the heterogeneity of the reservoir-fractures with various dip angles cut each other to form a complex networked fracture system. The water channelling problem is serious. A large amount of the remaining oil has been sealed in the matrix. The water flooding direction is complicated and severely reduces oil production. Fracture is the main factor that affects the effect of reservoir water flooding. Improving the swept area of fractured reservoirs is the direction of water flooding development and adjustment.

(3) The water flooding features and effects of fractured reservoirs with different fracture scales are different. When the fracture opening size reaches the mediumsmall scale or above, the water channelling will affect oil development. Water injection is invalid.

(4) The application of flexible gel to block large-scale fractures, nano-microspheres to block small-medium-scale fractures, and surfactants to change the wettability of microscale pores has been promoted in oilfields. It shows that this technology can effectively improve the effect of water injection and increase oilfield recovery. The application prospects of the technology are abroad, which can provide a technical basis for the comprehensive treatment of oil stabilization and water control in complex carbonate oil and gas fields at home and abroad.

\section{Data Availability}

The data used to support the findings of this study are included within the article.

\section{Conflicts of Interest}

The authors declare that there are no conflicts of interest regarding the publication of this paper.

\section{References}

[1] B. Zhang and J. Liu, "Classification and characteristics of karst reservoirs in China and related theories," Petroleum Exploration and Development, vol. 36, no. 1, pp. 19-36, 2009.

[2] S. J. Mazzullo and G. V. Chilingarian, "Chapter 9 Hydrocarbon reservoirs in karsted carbonate rocks," Developments in Petroleum Science, vol. 44, pp. 797-865, 1996.

[3] Y. Liu, Z. Tian, and R. Guo, "Review and prospective of rocktyping for complex carbonate reservoirs," Progress in Geophysics, vol. 32, no. 5, pp. 2057-2064, 2017.

[4] R. P. Major and M. H. Holtz, "A new meshless local PetrovGalerkin (MLPG) approach in computational mechanics," Computational Mechanics, vol. 22, pp. 117-127, 1990.

[5] Y. Li, Z. Kang, Z. Xue, and S. Zheng, "Theories and practices of carbonate reservoirs development in China," Petroleum Exploration and Development, vol. 45, no. 4, pp. 669-678, 2018.

[6] Z. Jin, X. Tan, and R. Guo, "Pore structure characteristics and control factors of carbonate reservoirs: the cretaceous Mishrif formation, halfaya oilfield, Iraq," Acta Sedimentologica Sinica, vol. 36, no. 5, pp. 981-994, 1998.

[7] Y. Liu, B. Liu, and D. Liu, "Analytic model of single well production performance in triple porosity medium reservoirs," Journal of Oil and Gas Technology, vol. 33, no. 2, pp. 123-127, 2011.

[8] Y. Li, Y. Hu, and B. Li, "Influencing factors of deliverability of horizontal wells in carbonate gas condensate reservoirs," Petroleum Geology and Recovery Efficiency, vol. 16, no. 2, pp. 85-87+116-117, 2009.

[9] W. Zhao, L. Zhao, and X. Wang, "Phase behavior characteristics and water-flooding development technical policy of weakly volatile oil in carbonate reservoirs," Petroleum Exploration and Development, vol. 43, no. 2, pp. 281-286, 2016.

[10] Q. Yang, J. Zhang, and T. Hao, "Seismic stepped prediction methods of sub-salt carbonate reservoirs in pre-caspian basin," Natural Gas Geoscience, vol. 25, no. 8, pp. 1216-1266, 2014.

[11] L. He, L. Zhao, and J. Liu, "Complex relationship between porosity and permeability of carbonate reservoirs and its controlling factors: a case of platform facies in pre-caspian basin," Petroleum Exploration and Development, vol. 41, no. 2, pp. 206-214, 2014.

[12] J. Zhang, "Seismic stepped prediction methods of sub-salt carbonate reservoirs in pre-caspian basin," Marine Geology Frontiers, vol. 27, no. 7, pp. 50-56, 2011.

[13] L. Liu, Y. Zhu, and A. Hu, "Petroleum geology of pre-salt sediments in pre-caspian basin," Journal of Southwest Petroleum Institute, vol. 24, no. 3, pp. 11-15, 2002.

[14] S. Wang, L. Zhao, X. Cheng, Z. Fan, and L. He, "Geochemical characteristics and genetic model of dolomite reservoirs in the eastern margin of the pre-caspian basin," Petroleum Science, vol. 9, no. 2, pp. 161-169, 2012.

[15] C. Yu, L. Mi, and C. Wang, "Percolation characteristics investigation of microscopic remaining oil in water flooding 
reservoir with ultra-high water cut," Fault-block Oil \& Gas Field, vol. 23, no. 05, pp. 592-594+598, 2016.

[16] J. Li, H. Sun, and H. Jiang, "Application of microfluidic models in oil and gas field development," Petroleum Science Bulletin, vol. 3, no. 03, pp. 284-301, 2018.

[17] H. J. Vogel, K. Roth, and S. Shen, "Quantitative morphology and network representation of soil pore structure," Advances in Water Resources, vol. 24, no. 3, pp. 233-242, 2001.

[18] J. Li, Y. Liu, and Y. Gao, "Effects of microscopic pore structure heterogeneity on the distribution and morphology of remaining oil," Petroleum Exploration and Development, vol. 45, no. 6, pp. 1043-1052, 2018.

[19] G. Cheng, J. Yin, and Y. Liu, "3D digital rock core reconstruction based on casting slice image," Science Technology and Engineering, vol. 15, no. 18, pp. 16-21, 2015.

[20] J. Yao, X. Zhao, and Y. Yi, “The current situation and prospect on digital core technology," Petroleum Geology and Recovery Efficiency, vol. 12, no. 6, pp. 52-54, 2005.

[21] X. Zhao, J. Yao, and J. Tao, "A method of constructing digital core by simulated annealing algorithm," Applied Mathematics-A Journal of Chinese Universities Series A, vol. 22, no. 2, pp. 127-133, 2007.

[22] Y. Zhu, G. Tao, and W. Fang, "Application of image processing technique in digital core modeling," Journal of Oil and Gas Technology, vol. 29, no. 5, pp. 54-57, 2007.

[23] I. Verri, A. Della Torre, G. Montenegro et al., "Development of a digital rock physics workflow for the analysis of sandstones and tight rocks," Journal of Petroleum Science and Engineering, vol. 156, pp. 790-800, 2017. 